\title{
Investigating genetic control of pigmentation in mutant barley lines with RNA-seq
}

\author{
N. Shmakov ${ }^{1 *}$, A. Glagoleva ${ }^{1,2}$, A. Doroshkov ${ }^{1}$, G. Vasiliev$^{1}$, N. Shatskaya ${ }^{1}$, \\ O. Shoeva ${ }^{1}$, D. Afonnikov ${ }^{1,2}$, E. Khlestkina ${ }^{1}$ \\ ${ }^{1}$ Institute of Cytology and Genetics SB RAS, Novosibirsk, Russia \\ ${ }^{2}$ Novosibirsk State University, Novosibirsk, Russia \\ *e-mail:shmakov@bionet.nsc.ru
}

Key words: RNA-seq, transcriptomics, barley, de novo assembly, differential expression

Motivation and Aim: Plant pigments are compounds that colorize plant organs and tissues. The most important plant pigment is chlorophyll that takes part in photosynthesis - the process that impacts all life on earth. Phytomelanin is ather plant pigments that gives black coloration to certain plant organs. Phytomelanin containing barley lines are believed to be more resistant to fungal pathogens [1].

Plant nearly-isogenic lines (NILs) with abnormal pigmentation are an appropriate object to investigate genetic machinery behind plant pigments synthesis and distribution. Considering genetic similarity of NILs, RNA-seq method could be implemented to specifically discover genes responsible for plant pigmentation control.

Methods and Algorithms: Total RNA was extracted from plants of Hordeum vulgare (barley) NILs Bowman, i:BwAlm with partial chlorophyll deficiency and BLP with partial melanism. Sequencing was carried out with IonTorrent platform. Libraries were filtered and quality-controlled with Prinseq tool. De novo assembly was performed using Trinity software. Assembled contigs were then aligned to $H$. vulgare reference genome. Fragments that showed no significant homology to the genome were functionally annotated using TransDecoder and Trinnotate tools.

Libraries were mapped to the reference genome using TopHat2 and STAR software. Differential expression was assessed with Cufflinks pipeline and EdgeR package. DEGs were functionally analyzed with AgriGO and PlantCyc databases.

Differential expression of a list of genes was verified with RT-qPCR. Expression of novel assembled genes was verified with PCR.

Results: Novel transcripts and lists of DEGs were discovered for each of the studied NILs. Genes up-regulated in i:BwAlm line are involved in vesicular transport, while genes up-regulated in BLP line participate in several metabolic pathways, including cutin and suberin monomers biosynthesis. Novel genes predicted with de novo assembly include several disease resistance genes for line $\mathrm{i}: \mathrm{Bw} A l m$ and autophagy-related gene for line BLP.

Conclusions: This work provides new insight on genetic mechanisms underlying partial albinism and melanism phenotypes formation in barley NILs. Our results demonstrate interaction between chloroplast and nuclear genomes in $\mathrm{i}: \mathrm{Bw}$ Alm line and activation of several metabolic pathways in BLP line. Finally, we predict several genes not encountered in $H$. vulgare before.

Acknowledgements: The work is supported by the RSF (16-16-04073).

\section{References}

1. Zhou X., Chao M., Liang X. (1991) Screening and testing of barley varieties for scab resistance (in Chinese with English abstract). Acta Phytophylacica Sinica. 18:261-265. 